University of South Carolina

Scholar Commons

10-26-2009

\title{
Wavepacket Approach to the Cumulative Reaction Probability within the Flux Operator Formalism
}

Sophya Garashchuk

University of South Carolina--Columbia, sgarashc@chem.sc.edu

Tijo Vazhappilly

University of South Carolina - Columbia

Follow this and additional works at: https://scholarcommons.sc.edu/chem_facpub

Part of the Chemistry Commons

Publication Info

Published in Journal of Chemical Physics, Volume 131, 2009, pages 164108-.

(c) Journal of Chemical Physics 2009, American Institute of Physics

This Article is brought to you by the Chemistry and Biochemistry, Department of at Scholar Commons. It has been accepted for inclusion in Faculty Publications by an authorized administrator of Scholar Commons. For more information, please contact digres@mailbox.sc.edu. 


\title{
Wavepacket approach to the cumulative reaction probability within the flux operator formalism
}

\author{
Sophya Garashchuk ${ }^{\mathrm{a})}$ and Tijo Vazhappilly \\ Department of Chemistry and Biochemistry, University of South Carolina, \\ Columbia, South Carolina 29208, USA
}

(Received 10 July 2009; accepted 30 September 2009; published online 26 October 2009)

\begin{abstract}
Expressions for the singular flux operator eigenfunctions and eigenvalues are given in terms of the Dirac $\delta$-function representable as a localized Gaussian wavepacket. This functional form enables computation of the cumulative reaction probability $N(E)$ from the wavepacket time-correlation functions. The Gaussian based form of the flux eigenfunctions, which is not tied to a finite basis of a quantum-mechanical calculation, is particularly useful for approximate calculation of $N(E)$ with the trajectory based wavepacket propagation techniques. Numerical illustration is given for the Eckart barrier using the conventional quantum-mechanical propagation and the quantum trajectory dynamics with the approximate quantum potential. $N(E)$ converges with respect to the Gaussian width parameter, and the convergence is faster at low energy. The approximate trajectory calculation overestimates tunneling in the low energy regime, but gives a significant improvement over the parabolic estimate of the tunneling probability. (C) 2009 American Institute of Physics.
\end{abstract}

[doi:10.1063/1.3251333]

\section{INTRODUCTION}

Quantum-mechanical (QM) theoretical studies of thermal reaction rate constants $k(T)$ and cumulative reaction probabilities $N(E)$ are based on the popular trace expressions of Miller et al. ${ }^{1}$ which involve the symmetrized flux operator

$$
\begin{aligned}
& \bar{F}=\frac{l}{2 m}\left[\hat{H}, \theta\left(x-x_{0}\right)\right], \\
& \bar{F}=\frac{l}{2 m}\left[\hat{p}, \delta\left(x-x_{0}\right)\right],
\end{aligned}
$$

where $\theta\left(x-x_{0}\right)$ is the Heaviside function and $\delta\left(x-x_{0}\right)$ is the Dirac $\delta$-function; $x$ is the reaction coordinate, $m$ is the reduced mass conjugate to $x$, and $x_{0}$ is a location of the surface dividing reactants and products. Two flux operators enter the expression for $N(E)$, which in atomic units is

$$
N(E)=2 \pi^{2} \operatorname{Tr}[\bar{F} \delta(E-\hat{H}) \bar{F} \delta(E-\hat{H})],
$$

and the thermal reaction rate constant expression

$$
k(T) Q(T)=\int_{0}^{\infty} C_{f f}(t) d t
$$

in terms of the flux-flux correlation function $C_{f f}(t)$,

$$
C_{f f}(t)=\operatorname{Tr}\left[e^{-\beta \hat{H} / 2} \bar{F} e^{-\beta \hat{H} / 2} e^{\imath \hat{H} t} \bar{F} e^{-l \hat{H} t}\right] .
$$

In the equations above $T$ labels temperature and $Q(T)$ is the reactant partition function. Variable $\beta=1 /\left(k_{B} T\right)$ is the thermal evolution variable. The real time is labeled $t$. Locations of the dividing surfaces where the flux is computed will be taken the same and set to $x_{0}=0$ below.

\footnotetext{
${ }^{a)}$ Electronic mail: sgarashc@mail.chem.sc.edu.
}

For both quantities, $N(E)$ and $k(T)$, the dynamical part of the calculation involves evaluation of the trace of the operators. $N(E)$ is a more general quantity from which the reaction rate constants can be computed for any temperature,

$$
k(T) Q(T)=(2 \pi)^{-1} \int_{-\infty}^{\infty} e^{-\beta E} N(E) d E,
$$

but $C_{f f}(t)$ is better suited for numerical evaluation with conventional $\mathrm{QM}$ methods, such as propagation in complex time, ${ }^{2}$ due to the damping effect of the Boltzmann operator $\exp (-\beta \hat{H})$ on the wave function.

In the early finite basis implementation Light and Park $^{2}$ empirically discovered that $\bar{F}$ given by Eq. (2) has only two nonzero eigenvalues, which greatly reduces computational cost of the trace evaluation. The two eigenvalues are negatives of each other and the corresponding eigenvectors are complex conjugate of each other. The eigenvalues and eigenfunctions were computed by diagonalizing the flux operator matrix. Later Seideman and Miller ${ }^{3}$ derived eigensolutions in terms of basis functions and showed that the absolute value of nonzero eigenvalues increases (to infinity) with increase in the basis size. The low rank of $\bar{F}$ is central to the quantum transition state wavepacket method of Light and Zhang, ${ }^{4,5}$ enabling efficient calculations of $N(E)$ for tetratomic systems. Approximate trajectory-based implementations of the trace formulas, however, could not take advantage of the low rank of $\bar{F}$ because the eigenfunctions of $\bar{F}$ defined in a finite basis typically exhibit oscillations extended in coordinate space and, therefore, are not well suited for trajectory representation. Semiclassical implementations with trajectories were focused on evaluation of the trace in a complete set of the position operator states, generally leading to expensive double phase space integrals., 
In Sec. II we generalize the finite basis solution to analytical expressions in terms of the Dirac $\delta$-function, which can be represented as a limiting form of nonsingular functions, such as a Gaussian function. In Sec. III we illustrate the properties of the solution by computing cumulative reaction probabilities for the Eckart barrier using QM splitoperator propagator ${ }^{8}$ and trajectory dynamics with the approximate quantum potential (AQP). ${ }^{9}$ Section IV concludes.

\section{WAVEPACKET FORMULATION FOR $N(E)$ WITHIN THE FLUX OPERATOR FORMALISM}

Using the low rank of the flux operator, the cumulative reaction probability given by Eq. (3) can be compactly expressed in terms of the wave function time-correlation functions. The flux operator forms of Eqs. (1) and (2) are equivalent in the complete basis limit, but only the latter expression gives just two nonzero eigenvalues in a finite basis and will be considered below for a one-dimensional system. Multidimensional generalization can be achieved by using a basis, which is a direct product of the one-dimensional basis in the reaction coordinate and the real orthogonal basis for the remaining coordinates. ${ }^{4}$ As have been observed in Ref. 2, in a one-dimensional real basis the nonzero eigenvalues $\pm \lambda$ $(\lambda>0)$ and the corresponding eigenvectors are related to each other,

$$
\bar{F}\left|\phi^{ \pm}\right\rangle= \pm \lambda\left|\phi^{ \pm}\right\rangle, \quad\left|\phi^{+}\right\rangle^{*}=\left|\phi^{-}\right\rangle .
$$

Substituting the spectral representation of the flux operator

$$
\bar{F}=\lambda\left(\left|\phi^{+}\right\rangle\left\langle\phi^{+}|-| \phi^{-}\right\rangle\left\langle\phi^{-}\right|\right)
$$

into Eq. (3), using the property (7) and the integral form of the $\delta$-function,

$$
2 \pi \delta(E-\hat{H})=\int_{-\infty}^{\infty} e^{-l \hat{H} t} e^{l E t} d t
$$

one obtains $N(E)$ in terms of the Fourier transforms of the wavepacket correlation functions,

$$
\begin{aligned}
N(E)= & \lambda^{2}\left(\left|\int_{-\infty}^{\infty}\left\langle\phi^{+}(0) \mid \phi^{+}(t)\right\rangle e^{\imath E t} d t\right|^{2}\right. \\
& \left.-\left|\int_{-\infty}^{\infty}\left\langle\phi^{+}(0) \mid \phi^{-}(t)\right\rangle e^{\imath E t} d t\right|^{2}\right) .
\end{aligned}
$$

The eigenvectors and eigenvalues of the flux operator can be straightforwardly determined in an arbitrary coordinate space basis $\vec{f}(x)$ of the size $N_{b}$ by constructing the (Hermitian) matrix $\mathbf{F}$,

$$
F_{i j}=\left\langle f_{i}|\bar{F}| f_{j}\right\rangle
$$

and finding its eigenvectors with nonzero eigenvalues,

$$
\mathbf{F} \vec{c}=\lambda \vec{c} .
$$

As was shown by Seideman and Miller ${ }^{3}$ these eigensolutions can be determined without the matrix diagonalization: For the basis of even/odd parity with respect to $x_{0}=0$ the eigenvalues are

$$
\lambda^{ \pm}= \pm \frac{1}{2 m}\|\vec{f}(0)\| \cdot\left\|\vec{f}^{\prime}(0)\right\| .
$$

The corresponding eigenvectors are

$$
\vec{c}^{ \pm}=\frac{1}{\sqrt{2}} \cdot\left(\frac{\vec{f}(0)}{\|\vec{f}(0)\|} \pm \imath \frac{\vec{f}^{\prime}(0)}{\left\|\vec{f}^{\prime}(0)\right\|}\right) .
$$

Prime denotes differentiation with respect to $x$.

The eigensolutions of $\bar{F}$ given by Eqs. (13) and (14) are singular and do not converge with respect to the basis size. However, the coordinate space eigenfunctions $\phi^{ \pm}(x)$ $=\vec{f}(x) \cdot \vec{c}$,

$$
\phi^{ \pm}(x)=\frac{1}{\sqrt{2}}\left(\frac{\sum_{n} f_{n}(x) f_{n}(0)}{\sum_{n} f_{n}(0)^{2}}+l \frac{\sum_{n} f_{n}(x) f_{n}^{\prime}(0)}{\sum_{n} f_{n}^{\prime}(0)^{2}}\right)
$$

are suggestive of a general functional form that does not employ coordinate space bases or grids. Equations (13) and (15) can be rewritten in terms of the $\delta$-function (which is even with respect to $x_{0}=0$ ) using its finite basis representation $\delta(x-y)=\Sigma_{n} f_{n}(x) f_{n}(y)$,

$$
\begin{aligned}
& \delta(x)=\left.\delta(x-y)\right|_{y=0}=\sum_{n} f_{n}(x) f_{n}(0), \\
& \frac{d}{d x} \delta(x)=-\left.\frac{d}{d y} \delta(x-y)\right|_{y=0}=-\sum_{n} f_{n}(x) f_{n}^{\prime}(0) .
\end{aligned}
$$

The numerators in Eq. (15) can be expressed in integral form as

$$
\sum_{n} f_{n}(0)^{2}=\int \sum_{n} f_{n}(x) f_{n}(0) \delta(x) d x=\int \delta(x)^{2} d x=\langle\delta \mid \delta\rangle,
$$

and, using integration by parts and Eq. (17),

$$
\begin{aligned}
\sum_{n} f_{n}^{\prime}(0)^{2} & =\sum_{n} f_{n}^{\prime}(0) \int f_{n}^{\prime}(x) \delta(x) d x \\
& =-\int \sum_{n} f_{n}^{\prime}(0) f_{n}(x) \delta^{\prime}(x) d x \\
& =\int \delta^{\prime}(x)^{2} d x .
\end{aligned}
$$

Then, the eigenfunctions given by Eq. (15) can be written as

$$
\phi^{ \pm}(x)=\frac{\delta(x)}{\sqrt{2\langle\delta \mid \delta\rangle}} \mp \frac{\imath \delta^{\prime}(x)}{\sqrt{2\left\langle\delta^{\prime} \mid \delta^{\prime}\right\rangle}},
$$

and the corresponding eigenvalues, Eq. (13), as

$$
\lambda^{ \pm}= \pm \frac{\sqrt{\langle\delta \mid \delta\rangle\left\langle\delta^{\prime} \mid \delta^{\prime}\right\rangle}}{2 m} .
$$

In principle, the limit of any $\delta$-function sequence ${ }^{10}$ can be used in Eqs. (20) and (21). For example, expressions of Matzkies and Manthe ${ }^{11}$ derived for the momentum limited flux in the context of the basis or grid wave function representation are consistent with Eqs. (20) and (21) if the $\delta$-function is represented as a limit of the sinc function 
sequence. ${ }^{10}$ For the trajectory-based propagation methods, ${ }^{9,12-16}$ however, the Gaussian form of the $\delta$-function

$$
\delta(x)=\lim _{\gamma_{0} \rightarrow \infty} \sqrt{\frac{\gamma_{0}}{\pi}} \exp \left(-\gamma_{0} x^{2}\right)
$$

is especially useful because these methods are exact for Gaussian wavepackets, and because localized in coordinate space initial wave function can be efficiently sampled with trajectories. The normalized flux operator eigenvectors become

$$
\phi^{ \pm}(x, 0)=\left(\frac{2 \gamma_{0}}{\pi}\right)^{1 / 4} \exp \left(-\gamma_{0} x^{2}\right)\left(\frac{1}{\sqrt{2}} \pm \imath \sqrt{2 \gamma_{0}} x\right)
$$

with the corresponding eigenvalues

$$
\lambda^{ \pm}= \pm \frac{\gamma_{0}}{m \sqrt{8 \pi}} .
$$

The eigenvalues and the eigenfunctions do not converge with respect to $\gamma_{0}$. At the same time the wave function variance multiplied by the eigenvalue is a constant, $\lambda\left\langle x^{2}\right\rangle$ $=1 /(m \sqrt{32 \pi})$.

At the beginning of time evolution $t=0$, the initial wave function given by Eq. (23) is highly localized (and $\lambda$ infinitely large as $\gamma_{0} \rightarrow \infty$ ) and, consequently, delocalizes on a short time scale. Propagation of highly localized wavepackets is numerically challenging, requiring very short time step and, for QM propagation, dense grids, but for a short time the propagation can be performed analytically in the parabolic approximation $V_{\text {par }}$ to the potential. Assuming that the dividing surface is located at the top of a potential barrier $V$,

$$
V \approx V_{\mathrm{par}}=V(0)-\frac{m w^{2} x^{2}}{2},
$$

and denoting the width of a "coherent" Gaussian wavepacket $\gamma_{\text {coh }}$,

$$
\gamma_{\mathrm{coh}}=\frac{m w}{2},
$$

in the spirit of Heller's expressions for evolving Gaussian, ${ }^{12}$ the time dependence of the Gaussian-based flux eigenfunction is

$$
\begin{aligned}
& \phi^{+}(x, t)=\left(\frac{2 \gamma_{0}}{\pi}\right)^{1 / 4} \alpha_{t} \exp \left(-\gamma_{t} x^{2}-\imath V_{0} t\right)\left(1+4 l \sqrt{\gamma_{0}} \alpha_{t}^{2} x\right), \\
& \gamma_{t}=\frac{\gamma_{0} \cosh w t-\imath \gamma_{\mathrm{coh}} \sinh w t}{\cosh w t+l\left(\gamma_{0} / \gamma_{\mathrm{coh}}\right) \sinh w t} \\
& \alpha_{t}=\frac{1}{\sqrt{2}}\left(\cosh w t+\frac{l \gamma_{0}}{\gamma_{\mathrm{coh}}} \sinh w t\right)^{-1 / 2}
\end{aligned}
$$

Duration of the analytical propagation $t_{a}$ can be estimated from the deviation between the gradients of the full and parabolic potentials $\Delta\left(t_{a}\right)$,
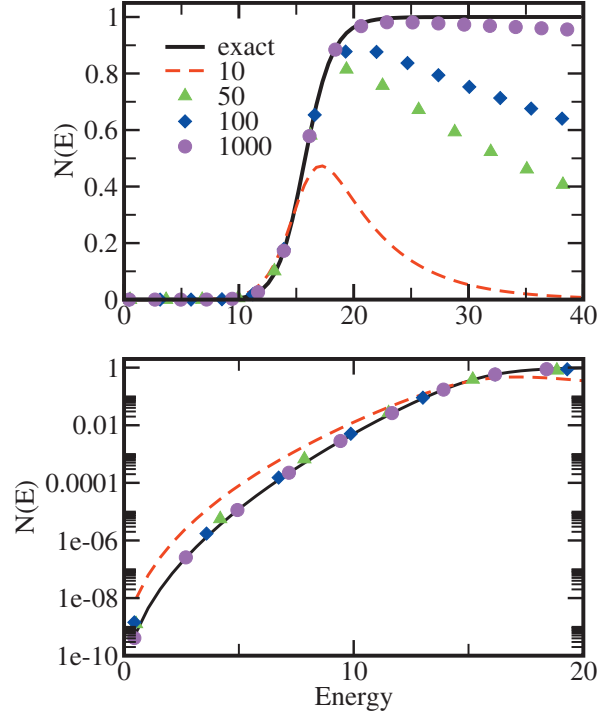

FIG. 1. Convergence of the reaction probability $N(E)$ for the Eckart barrier with respect to the initial Gaussian width parameter $\gamma_{0}$. The results are converged with respect to the final propagation time. Values of $\gamma_{0}$ in $\mathrm{a}_{0}^{-2}$ are marked on the legend. Low energy probabilities are shown on a logarithmic scale on the lower panel. (Not all of the resolved energy values are marked with symbols.)

$$
\Delta\left(t_{a}\right)=\frac{\left\langle\phi^{+}\left(t_{a}\right)\left|\left(V^{\prime}-V_{\mathrm{par}}^{\prime}\right)^{2}\right| \phi^{+}\left(t_{a}\right)\right\rangle}{\left\langle\phi^{+}\left(t_{a}\right)\left|\left(V^{\prime}\right)^{2}\right| \phi^{+}\left(t_{a}\right)\right\rangle} .
$$

Analytical short evolution with the Boltzmann operator has been employed by Thachuk and Schatz ${ }^{17}$ in the context of thermal reaction rate calculations to regularize a singular initial $\delta$-function.

\section{IMPLEMENTATION AND DISCUSSION}

\section{A. Quantum-mechanical implementation}

The wavepacket formulation given by Eqs. (10), (23), and (24) is illustrated by computing the reaction probability $N(E)$ for the standard one-dimensional model of the $\mathrm{H}_{3}$ transition state (for example, Ref. 18), i.e., the Eckart barrier scaled to have $m=1$,

$$
V=\frac{D}{\cosh ^{2} a x} \text {. }
$$

In scaled units the barrier height is $D=16$ and $a=1.3624 \mathrm{a}_{0}^{-1}$. The standard split-operator method ${ }^{8}$ is employed to propagate a wavepacket represented on a spatial grid (equivalent to the sine basis ${ }^{19}$ ). The relation given by Eq. (7) allows one to obtain the two correlation functions in Eq. (10) by propagating only $\phi^{+}(x, 0)$ and only forward in time. $N(E)$ is obtained from the correlation functions computed up to $t=3.0$, which is sufficiently long to obtain converged probabilities above $10^{-9}$. Figure 1 shows $N(E)$ for several large values of the initial Gaussian width parameter $\gamma_{0}$. The coherent width of the parabolic approximation to $V$ given by Eqs. (25) and (26) is $\gamma_{\text {coh }}=3.85 \mathrm{a}_{0}^{-2}$. Convergence of $N(E)$ at high energies $E>D$ is slow but monotonous. Convergence in the tunneling regime shown in Fig. 1(b) is much faster than for the high energies: calculation with the initial wavepacket width $\gamma_{0}$ 


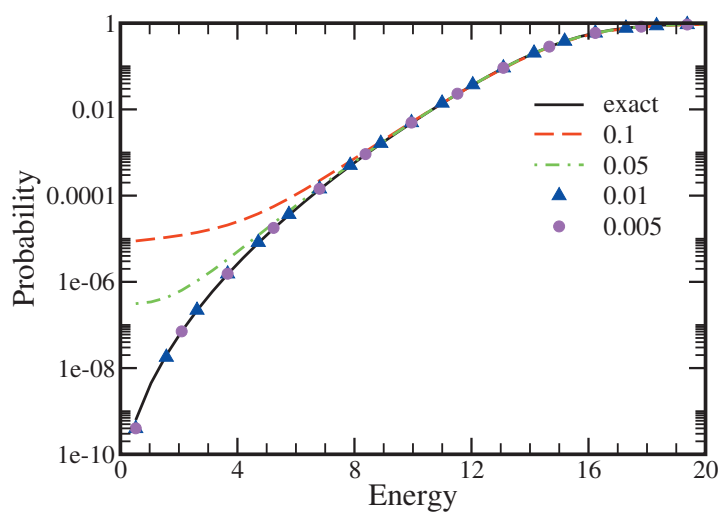

FIG. 2. The cumulative reaction probability obtained using QM propagation of the initial wave function broadened by the short-time analytical propagation in the parabolic approximation to the potential according to Eqs. (27)-(29) and (32). The results are compared with the exact analytical result for the Eckart barrier. Duration of the analytical propagation is marked on the legend. The results are converged with respect to the final propagation time, which is $t=3.0$

$=100 \mathrm{a}_{0}^{-2}$ already gives accurate results for energies below the barrier top.

The flux eigenvectors constructed in the basis representation are delocalized in space and, therefore, certain "regularization" of the wave function has to be included in QM propagation. Most of the numerical work involving flux eigenfunction propagation has been done for the thermal reaction rate constants, given by Eq. (4) and related formulations, because eigenfunctions of the thermalized flux operator that includes the Boltzmann operators are nonsingular and converge with respect to the basis size. ${ }^{20}$ The Boltzmann-operator evolution (later compensated) has also been used to regularize the flux operator eigenfunctions in computations of the cumulative reaction probability. ${ }^{21-23}$ Use of the momentum limited flux ${ }^{11}$ and absorbing potential ${ }^{3,24}$ are other regularization strategies employed with the basis or grid propagation methods.

Here the proposed Gaussian-based flux eigenfunction (23) is initially highly localized but delocalizes rapidly; the short time dependence of the real part of the Gaussian width parameter is $\mathfrak{R}\left(\gamma_{t}\right)=\gamma_{0}\left(1-\left(2 \gamma_{0} t / m\right)^{2}\right)$. This rapid delocalization, entailing very large grids and small time steps, can be reduced to allow cheaper numerical implementation: The initial wavepacket can be broadened by including analytical short-time propagation in the parabolic approximation to the barrier into the bra and ket of the correlation function

$$
C^{ \pm}(t)=\left\langle\phi^{ \pm}\left|e^{-l \hat{H} t}\right| \phi^{+}\right\rangle=\left\langle\phi^{ \pm}\left(t_{a}\right)\left|e^{-l \hat{H} t}\right| \phi^{+}\left(t_{a}\right)\right\rangle .
$$

For example, analytical propagation up to $t_{a}=0.005$ reduces the width of the initial Gaussian $\gamma_{0}=500 \mathrm{a}_{0}^{-2}$ by a factor of 25 . Convergence of $N(E)$ with respect to the analytical propagation time $t_{a}$ has to be tested and will depend on the desired accuracy. For the current application we find that convergence for $N(E) \geq 10^{-8}$ is achieved if the analytical propagation error $\Delta\left(t_{a}\right)$ defined by Eq. (30) is on the order of $1 \%$. Figure 2 shows the dependence of $N(E)$ on the analytical time propagation: There is a regime of analytical propagation times that are long enough to regularize the initial wavepacket without compromising accuracy of $N(E)$.

\section{B. Approximate quantum trajectory implementation}

The Gaussian form of the flux operator eigenfunctions (23) is especially useful for computations of the cumulative reaction probability using approximate trajectory-based propagation methods, which reproduce exact Gaussian wavepacket dynamics, such as frozen and thawed Gaussian wavepacket methods, ${ }^{12,13}$ the Wigner method, ${ }^{14}$ the Herman-Kluk propagator, and other forms of semiclassical initial-value representation methods. ${ }^{15,16}$ Below we use the quantum trajectory dynamics with the $\mathrm{AQP},{ }^{9}$ which is developed to provide estimates of quantum effects in high-dimensional systems. In this section the Planck's constant is included explicitly to indicate the terms that vanish in the classical limit $\hbar \rightarrow 0$, and $\nabla=d / d x$ is used for compactness of notations.

\section{Quantum trajectory dynamics with approximate quantum potential}

The AQP method, ${ }^{9}$ outlined here in one dimension and in its simplest form, is based on the quantum trajectory formulation $^{25,26}$ of the conventional time-dependent Schrödinger equation (TDSE)

$$
\left(-\frac{\hbar^{2}}{2 m} \nabla^{2}+V\right) \psi(x, t)=\imath \hbar \frac{\partial}{\partial t} \psi(x, t) .
$$

The wave function expressed in polar form by the real amplitude $A(x, t)$ and phase $S(x, t)$,

$$
\psi(x, t)=A(x, t) e^{\imath S(x, t) / \hbar},
$$

is represented by an ensemble of trajectories characterized by positions $x_{t}$, momenta $p_{t}$, and action functions $S_{t}$. The trajectory momentum is identified with the gradient of the phase

$$
p(x, t)=\nabla S(x, t) .
$$

The quantum trajectories evolve according to Newton's laws of motion obtained by substituting Eq. (34) into Eq. (33),

$$
\begin{aligned}
& \frac{d x_{t}}{d t}=\frac{p_{t}}{m}, \quad \frac{d p_{t}}{d t}=-\nabla(V+U), \\
& \frac{d S_{t}}{d t}=\frac{p_{t}^{2}}{2 m}-(V+U) .
\end{aligned}
$$

Function $U$ denotes the quantum potential

$$
U=-\frac{\hbar^{2}}{2 m} \frac{\nabla^{2} A(x, t)}{A(x, t)},
$$

which vanishes in the classical limit $\hbar \rightarrow 0$ for nonsingular wave function amplitudes. The time-dependent wave function density $A^{2}(x, t)$ satisfies the same continuity equation as classical fluid, from which conservation of the probability within the volume element $\delta\left(x_{t}\right)$ associated with each trajectory - the trajectory "weight" $w\left(x_{t}\right)$-follows:

$$
w\left(x_{t}\right)=A^{2}\left(x_{t}\right) \delta x_{t}, \quad \frac{d w\left(x_{t}\right)}{d t}=0 .
$$

Therefore, in principle, $A(x, t)$ can be reconstructed from the positions and weights of the trajectories. 
The quantum trajectory formulation is equivalent to the TDSE (33) and the only differences between the quantum and the classical trajectory dynamics are the quantum potential and quantum force acting on the trajectories in addition to their classical counterparts. Quantum trajectories appeal to our classical intuition and are used for visualization and interpretation. Exact numerical implementation, however, is extremely challenging in the presence of quantum interference. Besides the obvious numerical difficulties of evaluating $U$ and its derivatives at or near the nodes $(A(x, t)=0)$, the trajectory dynamics is inherently unstable. (One should note, though, that quantum interference is generally quenched in high-dimensional systems.) A comprehensive overview of the quantum trajectory dynamics field is given in Ref. 27 .

Garashchuk and Rassolov ${ }^{9,28}$ used the quantum trajectory formulation to develop an approximate approach where the quantum potential is determined variationally, thus conserving energy of the trajectory ensemble, from the linearization of the nonclassical component $r(x, t)$ of the momentum operator

$$
r(x, t)=\frac{\nabla A(x, t)}{A(x, t)} .
$$

Approximating $r(x, t)$ in terms of the linear fitting function $\widetilde{r}(x, t)$, minimization of $\left\langle(r-\widetilde{r})^{2}\right\rangle$, has a solution in terms of the moments of the trajectory distribution

$$
r(x, t) \approx \widetilde{r}(x, t)=-\frac{x-\langle x\rangle_{t}}{2\left(\left\langle x^{2}\right\rangle_{t}-\langle x\rangle_{t}^{2}\right)} .
$$

Then, the AQP (labeled $\widetilde{U}$ ) and its gradient are determined analytically,

$$
\tilde{U}=-\frac{\hbar^{2}}{2 m}\left(\widetilde{r}^{2}(x, t)+\nabla \widetilde{r}(x, t)\right) .
$$

The method is exact for Gaussian wavepackets and describes wavepacket bifurcation, moderate tunneling, and zero-point energy. It is numerically cheap and stable due to the "meanfield-like" procedure of determining AQP: using Eq. (39) the expectation values in Eq. (41) are computed as sums over trajectories labeled with index $k$,

$$
\begin{aligned}
\langle q(x)\rangle_{t} & =\int q(x) A^{2}(x, t) d x \\
& =\int q\left(x_{t}\right) A^{2}\left(x_{t}\right) \delta x_{t} \\
& =\sum_{k} q\left(x_{t}^{(k)}\right) w^{(k)} .
\end{aligned}
$$

In fact, explicit knowledge of $A(x, t)$ is not needed for the AQP propagation and, in the current implementation, even for the calculation of the wavepacket correlation functions in Eq. (10).

\section{Wave function evolution in the mixed coordinate/ polar representation}

To compute $N(E)$ we will propagate the flux operator eigenfunctions (20), which are products of a Gaussian and a linear function of $x$, using a mixed coordinate/polar wave function representation introduced in Ref. 29,

$$
\phi(x, t)=\psi(x, t) \chi(x, t),
$$

where $\psi(x, 0)$ is a normalized Gaussian and $\chi(x, 0)$ is a polynomial. The polar part $\psi(x, t)$, represented in terms of the quantum trajectories, approximately solves the TDSE (33) with external potential $V$. The coordinate part $\chi(x, t)$ is represented in a small basis,

$$
\chi(x, t)=\vec{g}(x) \cdot \vec{a}(t) .
$$

The mixed representation is advantageous because the AQP of Eqs. (41) and (42) is exact for a Gaussian and because the quantum trajectories describing the entire wave function $\phi(x, t)$, which has a node, could be unstable. Substituting Eqs. (44) and (45) into the TDSE for $\phi(x, t)$, using the fact that $\psi(x, t)$ is a solution of Eq. (33), multiplying the resulting expression by $\psi^{*}(x, t) \vec{g}(x)$, and integrating over $x$ using integration by parts, we obtain the time-evolution equations for the coefficients $\vec{a}$,

$$
\iota\langle\vec{g} \otimes \vec{g}\rangle \frac{d \vec{a}}{d t}=\left(\frac{\left\langle\vec{g}^{\prime} \otimes \vec{g}^{\prime}\right\rangle}{2 m}-l \frac{\left\langle p \vec{g} \otimes \vec{g}^{\prime}\right\rangle}{m}\right) \vec{a} .
$$

All integrals include $|\psi(x, t)|^{2}$. In particular, the last term of Eq. (46) contains matrix elements involving trajectory momenta,

$$
\left\langle p \vec{g} \otimes \vec{g}^{\prime}\right\rangle_{i j}=\int_{-\infty}^{\infty}|\psi(x, t)|^{2} p(x) g_{i}(x) \frac{d}{d x} g_{j}(x) d x .
$$

The polar part $\psi(x, t)$ is described in terms of quantum trajectories, initiated with zero initial momenta at positions sampling the real Gaussian part of the eigenfunctions (23), which is the same for $\phi^{+}(x)$ and $\phi^{-}(x)$. Function $\chi(x, 0)$ is the polynomial part of $\phi(x, 0)$. If $\chi(x, t)$ is restricted to a linear form and the potential is symmetric, then $\langle x\rangle=0$ and $\langle p\rangle=0$, and the time dependence of $\vec{a}$ is particularly simple,

$$
a_{1}(t)=a_{1}(0), \quad a_{2}(t)=a_{2}(0) \exp \left(-\int_{0}^{t} \frac{\langle p x\rangle_{\tau}+l / 2}{m\left\langle x^{2}\right\rangle_{\tau}} d \tau\right) .
$$

The AQP and its gradient are obtained from Eqs. (41) and (42). The resulting trajectory dynamics is stable.

\section{Computation of correlation functions}

In addition to stable trajectory dynamics, the mixed representation given by Eq. (44) allows a straightforward evaluation of the correlation functions using trajectory weights without reconstructing the amplitude of $\psi(x, t)$, and a reduction of propagation time by a factor of 2 . In the timecorrelation function expression, the time evolution can be divided, for example, equally between $\left\langle\phi_{i}\right|$ and $\left|\phi_{j}\right\rangle$,

$$
\begin{aligned}
C(t) & =\left\langle\phi_{i}\left|e^{-l H t}\right| \phi_{j}\right\rangle \\
& =\left\langle e^{\imath H t / 2} \phi_{i} \mid e^{-l H t / 2} \phi_{j}\right\rangle \\
& =\left\langle\phi_{i}(-t / 2) \mid \phi_{j}(t / 2)\right\rangle .
\end{aligned}
$$

Time evolution into the negative times, i.e., into the "past," 

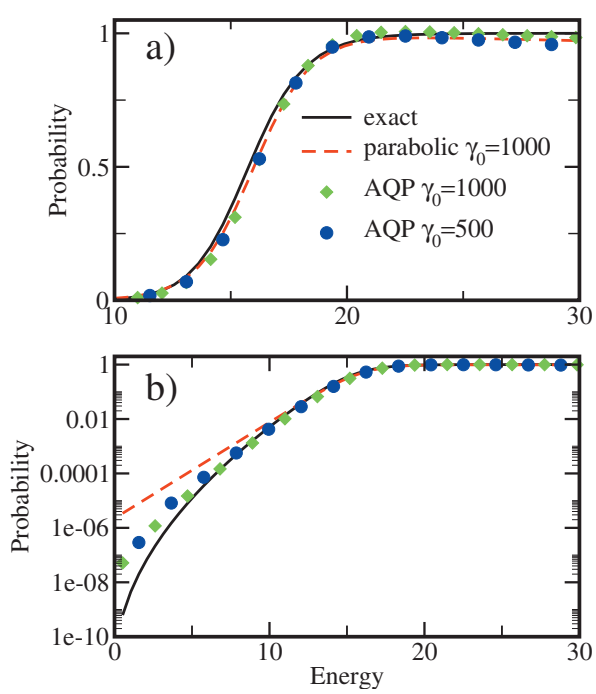

FIG. 3. The cumulative reaction probability obtained using the AQP trajectory dynamics for the Eckart barrier and using the analytical time-dependent wave function evolving in the parabolic potential approximating this barrier.

can be expressed as propagation of a complex conjugate wave function into positive times, i.e., into the "future,"

$\phi_{i}(-t)=e^{\imath H t} \phi_{i}(0)=\left(e^{-l H t} \phi_{i}^{*}(0)\right)^{*} \quad$ or $\quad\left(\phi_{i}(-t)\right)^{*}=\phi_{i}^{c}(t)$.

Superscript in $\phi_{i}^{c}$ indicates conjugation of $\phi_{i}$ at time $t=0$. For the mixed wave function given by Eq. (44), which has initially real polar part $\psi(x, 0)$, the advantages of splitting the time evolution between bra and ket are in the reduction of error due to approximations, and in computation of the correlation functions in terms of the trajectory weights without reconstruction of the wave function,

$$
\begin{aligned}
C(2 t) & =\int \phi_{i}^{c}(x, t) \phi_{j}(x, t) d x \\
& =\int \chi_{i}^{c}(x, t) \psi^{c}(x, t) \chi_{j}(x, t) \psi(x, t) d x \\
& =\int \chi_{i}^{c}(x, t) \chi_{j}(x, t) \psi^{2}(x, t) d x \\
& =\sum_{k} w_{k} e^{2 t S_{t}^{(k)}} \chi_{i}^{c}\left(x_{t}^{(k)}, t\right) \chi_{j}\left(x_{t}^{(k)}, t\right) .
\end{aligned}
$$

For the adopted linear form of $\chi(x, t)$,

$$
\phi^{ \pm}(x, t)=\psi(x, t)\left(a_{1}(t) \pm a_{2}(t) x\right),
$$

the correlation functions needed in Eq. (10) become

$$
C^{ \pm}(2 t)=\sum_{k} w_{k} e^{2 l S_{t}^{(k)}}\left(a_{1}(t)+a_{2}(t) x_{t}^{(k)}\right)\left(a_{1}(t) \mp a_{2}(t) x_{t}^{(k)}\right) .
$$

\section{Numerical results}

Figure 3 shows linear and logarithmic plots for the cumulative reaction probability $N(E)$ obtained for the initial Gaussian width of $\gamma_{0}=1000 \mathrm{a}_{0}^{-2}$ and $\gamma_{0}=500 \mathrm{a}_{0}^{-2}$. A short time analytical propagation was carried out up to $t_{a}=0.01$ for the
TABLE I. Timing and convergence of the moments for the AQP trajectory propagation. The relative errors are given at the final propagation time.

\begin{tabular}{cccc}
\hline \hline $\begin{array}{c}N_{\text {traj }} \\
\text { (thousands) }\end{array}$ & $\begin{array}{c}\text { Relative error } \\
\left\langle x^{2}\right\rangle\end{array}$ & $\begin{array}{c}\text { Relative error } \\
\langle x p\rangle\end{array}$ & $\begin{array}{c}\text { Relative time } \\
\text { per trajectory }\end{array}$ \\
\hline 0.5 & $3.9 \times 10^{-5}$ & $3.3 \times 10^{-5}$ & 1 \\
1 & $1.8 \times 10^{-5}$ & $1.6 \times 10^{-5}$ & 0.92 \\
5 & $2.6 \times 10^{-6}$ & $3.2 \times 10^{-6}$ & 0.85 \\
25 & $3.5 \times 10^{-7}$ & $6.3 \times 10^{-7}$ & 0.83 \\
50 & $1.3 \times 10^{-7}$ & $3.0 \times 10^{-7}$ & 0.83 \\
\hline
\end{tabular}

latter value of $\gamma_{0}$. Since propagation up to $t$ allows one to obtain correlation functions at $2 t$ [Eq. (52)], the trajectories are initialized at $t_{a} / 2=0.005$, as in the QM calculation in Sec. III A.

In this illustration our goal is to assess the AQP description of the tunneling probability; the numerical efficiency of the method has not been optimized. The trajectory positions are distributed uniformly with the weights and phases, respectively, defined by the real and imaginary components of $\gamma_{t}$ given by Eq. (28) for $t=t_{a / 2}$. The momenta are equal to the gradient of the phase [Eq. (35)]. For the linear representation of $\chi(x, t)$, the only quantities needed to determine the quantum force and the polynomial coefficient $a_{2}$ in Eq. (47) are $\left\langle x^{2}\right\rangle$ and $\langle p x\rangle$. Convergence of these quantities with respect to the number of trajectories is presented in Table I at the final propagation time $t=1.5$ for $\gamma_{0}=500 \mathrm{a}_{0}^{-2}$. Propagation of 1000 trajectories is sufficient to obtain relative errors of the moments below $2 \times 10^{-5}$, but we need on the order of $10^{6}$ trajectories to obtain the low tunneling probabilities with uniform sampling. In principle, the AQP parameters and coefficients $\vec{a}$ can be simply stored from a calculation with a few thousand trajectories and used to propagate larger number of independent trajectories, possibly with more effective trajectory sampling. The table also shows the relative timing per trajectory in the AQP dynamics for calculations with different numbers of trajectories. The total time scales linearly as in classical trajectory propagation. Approximate propagation techniques are usually slower than the conventional QM propagation for one- and two-dimensional systems, which is the case for this application. But in higher dimensionality, the cost of trajectory propagation scaling linearly with dimensionality will be cheaper than QM methods as, for example, in a three-dimensional application of Ref. 30.

As seen from Fig. 3, the AQP results are accurate for energies below the barrier up to tunneling probability of $10^{-5}$. The narrower initial wavepacket gives more accurate results. The AQP calculation overestimates tunneling below $10^{-5}$. Nevertheless, it is a significant improvement over the parabolic barrier approximation: For $\gamma_{0}=1000 \mathrm{a}_{0}^{-2}$, at the lowest computed energy, $E=0.52$, which is $3 \%$ of the barrier height, the parabolic barrier approximation overestimates tunneling by about $2 \times 10^{4}$, while the AQP value is 200 times smaller than that. In our implementation, there are two approximations: The first one is the linear quantum force and the second one is the linear form of $\chi(x, t)$. We attribute most of the error to the quantum force. Figure 4 shows the autocorrelation function of $\left|\phi^{+}\right\rangle$and the same for the initially 


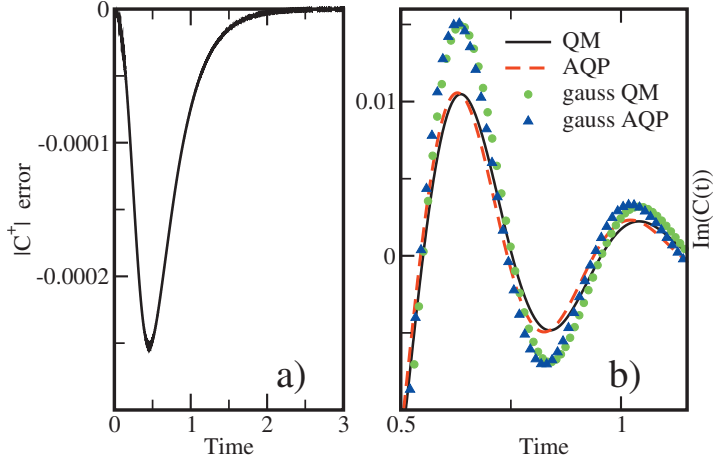

FIG. 4. Comparison of QM and AQP correlation functions. (a) Difference between the correlation function amplitudes obtained with $\mathrm{QM}$ and $\mathrm{AQP}$ propagations, $\left|C_{\mathrm{QM}}^{+}\right|-\left|C_{\mathrm{AQP}}^{+}\right|$. The relative error on the same time interval increases from $0 \%$ to $10 \%$. (b) Imaginary components of the correlation functions $C_{\mathrm{OM}}^{+}$and $C_{\mathrm{AOP}}^{+}$and of the autocorrelation function of the polar part of the wave function $\langle\phi(0) \mid \phi(t)\rangle$ are shown with lines and symbols, respectively. The correlation functions are normalized to 1 at $t=0$.

Gaussian part $C(t)=\langle\psi(x, 0) \mid \psi(x, t)\rangle$. Correlation functions in the figure shown for $\gamma_{0}=500 \mathrm{a}_{0}^{-2}$ were scaled to 1 at $t=0$. Small discrepancies in the phase between the QM and the AQP trajectory calculations are similar to those reported with the semiclassical dynamics. ${ }^{18,31}$ The AQP phase lags behind the QM phase, which affects the Fourier transforms of the correlation functions and the target quantity $N(E)$. Figure 4(a) shows the difference between the amplitudes for the QM and AQP correlation functions $C^{+}(t)$ in absolute sense. Its amplitude obtained with AQP is slightly lower than the QM value, while the relative error for the same time interval changes from $0 \%$ to $10 \%$. The difference for the Gaussian part of the wave function shows the same trend. Thus, the amplitude and the phase discrepancy can be attributed to underestimation of the "quantum energy" $U$ due to the linear form of the approximate quantum force.

\section{SUMMARY}

We have presented expressions for the symmetrized flux operator eigenvectors and eigenvalues in terms of the $\delta$-function, which can be defined as a limit of an analytical $\delta$-function sequence, such as a Gaussian function. ${ }^{10}$ This allows basis set independent spectral representation of the singular flux operator, which can be used with the trace expression for the cumulative reaction probability. The narrow (infinitely narrow in the $\delta$-function limit) Gaussian can be regularized for numerical work by the short-time analytical propagation in the parabolic approximation to the potential. While the Gaussian-based wavepacket approach is not necessarily the most convenient way to compute $N(E)$ with basis or grid QM time-evolution methods, the formulation is useful for nonbasis set propagation techniques such as trajectory-based approximate propagation methods.

Numerical example is given for the one-dimensional barrier using QM split-operator propagation on a grid and trajectory dynamics with the AQP with mixed coordinate/polar wave function representation. Reaction probability converges with respect to the initial Gaussian width and does so faster for low energies. The initial wavepacket can be broadened by short-time analytical evolution in the parabolic approximation to the barrier for numerical reasons. In the approximate quantum trajectory implementation, the mixed wave function representation allows for stable trajectory dynamics of the polar part; the wave function node is described by the polynomial coordinate part. In addition, the propagation time is reduced by a factor of 2 and the correlation functions are computed in a simple manner without the wave function reconstruction. The AQP-based implementation overestimated tunneling below $10^{-5}$ but gave a significant improvement on the parabolic barrier estimates.

The flux-eigenvector formulation for the energy-resolved cumulative reaction probabilities is sensitive to the accuracy of the wavepacket correlation functions, although it gives complete information about the system from which thermal reaction rate constants can be easily obtained [Eq. (6)]. Direct computation of the thermal rate constants, however, is expected to be more robust because the thermal averaging is done before wave function overlaps are analyzed. Approximate quantum trajectory implementation of the analytical flux eigensolutions for the thermal rate constants [Eq. (4)] and multidimensional applications will be reported in the future.

\section{ACKNOWLEDGMENTS}

This work was supported by the University of South Carolina.

${ }^{1}$ W. H. Miller, S. D. Schwartz, and J. W. Tromp, J. Chem. Phys. 79, 4889 (1983).

${ }^{2}$ T. J. Park and J. C. Light, J. Chem. Phys. 88, 4897 (1988).

${ }^{3}$ T. Seideman and W. H. Miller, J. Chem. Phys. 95, 1768 (1991).

${ }^{4}$ D. H. Zhang and J. C. Light, J. Chem. Phys. 104, 6184 (1996).

${ }^{5}$ J. C. Light and D. H. Zhang, Faraday Discuss. 110, 105 (1998).

${ }^{6}$ T. Yamamoto, H. Wang, and W. H. Miller, J. Chem. Phys. 116, 7335 (2002).

${ }^{7}$ C. Venkataraman and W. H. Miller, J. Chem. Phys. 126, 094104 (2007).

${ }^{8}$ M. D. Feit, J. A. Fleck, Jr., and A. Steiger, J. Comput. Phys. 47, 412 (1982).

${ }^{9}$ S. Garashchuk and V. A. Rassolov, J. Chem. Phys. 120, 1181 (2004).

${ }^{10}$ G. Arfken, Mathematical Methods for Physicists, 3rd ed. (Academic, New York, 1985).

${ }^{11}$ F. Matzkies and U. Manthe, J. Chem. Phys. 110, 88 (1999).

${ }^{12}$ E. J. Heller, J. Chem. Phys. 62, 1544 (1975).

${ }^{13}$ E. J. Heller, J. Chem. Phys. 75, 2923 (1981).

${ }^{14}$ R. C. Brown and E. J. Heller, J. Chem. Phys. 75, 186 (1981).

${ }^{15}$ M. F. Herman and E. Kluk, Chem. Phys. 91, 27 (1984).

${ }^{16}$ K. G. Kay, J. Chem. Phys. 100, 4377 (1994).

${ }^{17}$ M. Thachuk and G. C. Schatz, J. Chem. Phys. 97, 7297 (1992).

${ }^{18}$ F. Grossmann and E. J. Heller, Chem. Phys. Lett. 241, 45 (1995)

${ }^{19}$ D. T. Colbert and W. H. Miller, J. Chem. Phys. 96, 1982 (1992).

${ }^{20}$ E. Pollak, J. Chem. Phys. 107, 64 (1997).

${ }^{21}$ U. Manthe and F. Matzkies, Chem. Phys. Lett. 282, 442 (1998).

${ }^{22}$ F. Matzkies and U. Manthe, J. Chem. Phys. 108, 4828 (1998).

${ }^{23}$ F. Huarte-Larrañaga and U. Manthe, J. Phys. Chem. A 105, 2522 (2001).

${ }^{24}$ T. Seideman and W. H. Miller, J. Chem. Phys. 96, 4412 (1992).

${ }^{25}$ E. Madelung, Z. Phys. 40, 322 (1926).

${ }^{26}$ D. Bohm, Phys. Rev. 85, 166 (1952).

${ }^{27}$ R. E. Wyatt, Quantum Dynamics with Trajectories: Introduction to Quantum Hydrodynamics (Springer-Verlag, Berlin, 2005).

${ }^{28}$ S. Garashchuk and V. A. Rassolov, Chem. Phys. Lett. 376, 358 (2003)

${ }^{29}$ S. Garashchuk and V. A. Rassolov, J. Chem. Phys. 121, 8711 (2004).

${ }^{30}$ S. Garashchuk, V. A. Rassolov, and G. C. Schatz, J. Chem. Phys. 124, 244307 (2006).

${ }^{31}$ S. Garashchuk and V. A. Rassolov, J. Chem. Phys. 118, 2482 (2003). 